\title{
Novas Tecnologias em Ambientes de Aprendizagem; Estimulando o Aprender a Aprender, Transformando o Currículo e Ações
}

\author{
Jorge Ferreira Franco* \\ Roseli de Deus Lopes**
}

\section{Resumo}

Este texto apresenta experiências de aprendizagem através de uso cooperativo de tecnologias acessíveis da WEB com educadores e educandos na educação básica. O objetivo é estimular o conhecimento, a pesquisa, o entendimento e a aplicação contextualizada das novas tecnologias e instrumentos multimídia em ambientes de ensino aprendizagem, contribuindo para transformar a prática pedagógica e o currículo, incluindo o desenvolvimento da autonomia de aprender a aprender e a consciência da importância da educação continuada na vida dos indivíduos.

Palavras-chave: novas tecnologias, aprendizado cooperativo, currículo escolar, aprender a aprender, educação permanente

Title: New Technologies in Learning Environments; Stimulating the Learning to Learn, Transforming Curriculum and Actions

\begin{abstract}
This text presents learning experiences through cooperative use of accessible WEB technologies with educators and students at basic education. The aim is to stimulate new technologies and multimedia tools knowledge, research, understanding and context applications in learning environments, contributing for transforming pedagogical practice and curriculum, including the development of learning to learn autonomy and the awareness of lifelong learning importance for individuals' life.
\end{abstract}

Keywords: new technologies, cooperative learning, school curriculum, learning to learn, lifelong education

\section{Introdução}

A constante evolução dos sistemas de informação com base na fusão dos computadores e das telecomunicações (TICs), incluindo-se a ampliação do acesso aos recursos da WEB tem influenciado amplamente a vida dos indivíduos. Significando ser de fundamental importância para o desenvolvimento da sociedade de informação em que vivemos, preparar indivíduos que sejam capazes de compreender, utilizar e criar conhecimento fundamentado nos recursos propiciados pelas novas tecnologias (Franco, 2000).

Assim sendo, disseminar o conhecimento e uso das novas tecnologias em ambientes de ensino/aprendizagem pode contribuir efetivamente para transformar a educação, ampliar a comunicação entre a comunidade escolar, desenvolver o currículo-formação em uma perspectiva pós-moderna que possa gerar mais emancipação no aprender a aprender dos indivíduos de modo sustentável (Doll, 1997, Dolabela, 2003).

\footnotetext{
* Doutorando em Informática e Educação - Departamento de Sistemas Integráveis Poli / USP Professor Orientador de Informática Educativa - (POIE) - Prefeitura Municipal de São Paulo

*** Professora Doutora - Departamento de Sistemas Integráveis - Poli / USP - http://www.lsi.us.br/ roseli
} 
Estas perspectivas têm sido comprovadas conforme são realizados experimentos educacionais e ampliado o acesso dos Educadores e Educandos aos sistemas de informação e de comunicação, com utilização da convergência das TICs, teorias pedagógicas, formação de educadores via rede telemática e em local de atuação, aprendizagem por projetos, conceitos de empreendedorismo e técnicas da psicologia experimental em ambientes de ensino-aprendizagem (Franco, 2000a, Franco e Roseli, 2002, Dolabela, 2003, Valente, 2003).

As experiências educacionais que têm sido desenvolvidas na escola e no Laboratório de Sistemas Integráveis - LSI focam em apresentar e compartilhar com educadores e educandos conhecimento técnico e científico relativo às novas tecnologias, investigando e validando formas de propiciar mais estímulo e qualidade aos processos cognitivos/educativos dos indivíduos. A proposta é ativar estes processos com base em conceitos de sistemas de informação digitais, computação gráfica interativa, técnicas de Realidade Virtual e pesquisa, influenciando a formação de educandos/educadores, com tecnologia de ponta, desde a educação básica, de modo que possam compreender o que é o mundo na era da informação digital, e viver o digital entendendo um mundo educativo em multimídia capaz de favorecer o estudo/trabalho compartilhado em projetos colaborativos (Biazus, 2003). Embasam a aplicação do estado da arte da tecnologia na formação dos indivíduos a partir da educação básica projetos que estimulam a leitura, a escrita, e a aprendizagem colaborativa via meios eletrônicos, tais como o NICE - Narrative, Immersive, Constructionist/Collaborative Environments for Learning in Virtual Reality (Roussos et al., 1997), e o GARAGEM Digital ${ }^{\text {TM }}$ (Franco e Roseli, 2002, Pimenta, 2004).

Como resultado, as experiências descritas neste artigo mostram que tem havido incremento na aproximação e interação entre as pesquisas realizadas por instituições de ensino superior e a educação básica, crescimento na cooperação entre educadores e educandos, maior estímulo à pesquisa, aumento da autonomia no aprender a aprender, e despertar da consciência individual sobre a importância de uma educação continuada fundamentada na aquisição de conhecimento técnico e teórico de modo interdisciplinar, a fim de que o indivíduo possa compreender melhor a complexidade de suas investigações e transformá-las em produtos que beneficiem a sociedade.

\section{Influências Tecnológicas, Transformação Curricular e Social}

A base das ações tecnológicas está ancorada nos padrões acessíveis e não proprietários da Internet para propiciar acesso e desenvolvimento sustentável do conhecimento sobre as novas tecnologias que formam a rede mundial de computadores. Desta forma, estimase contribuir para o desenvolvimento de um currículo não-linear, interdisciplinar, beneficiando a comunidade escolar com as possibilidades de aprender através de comunicação interativa, para além do hipertexto em formato de áudio, vídeo e texto. Ou seja, usar com criatividade a convergência Internet/mídia para inovação nos negócios e na educação, tendo a Internet como resultado da apropriação social de sua tecnologia por seus usuários/produtores (Castells, 2003).

Novas tecnologias accessíveis têm influenciado mais e mais transformações nos negócios do mundo corporativo tanto pela questão de diminuição de custos relativos aos investimentos em software proprietário e flexibilidade de adaptação, quanto à facilidade 
de criação, escala, produção, manutenção de sistemas e possibilidades diversas de comunicação com o usuário. Por exemplo, via interfaces em duas e três dimensões com a combinação de linguagens padrão da WEB, tais como Hypertext Markup Language HTML (2D) e Virtual Reality Modeling Language - VRML (3D), JavaScript, JAVA, incluindo-se o uso de linguagem natural e simulações (Ames et. al, 1997, Perlin, 2004).

Utilizar tais tecnologias na educação básica pode apresentar conceitos técnicos/científicos fundamentados em sistemas de informação e computação gráfica para que as crianças de hoje tornem-se adultos capazes de aplicá-los com criatividade e inovação ao longo de suas vidas. Segundo Furth (1999) citado por Franco (2000) justifica-se empregar o estado da arte das tecnologias para aprimorar o ambiente escolar, pois talvez seja oportunidade única para muitas crianças acessarem e aprenderem como tais tecnologias podem melhorar a qualidade de suas vidas através do desenvolvimento de suas habilidades e competências para compreender e modificar o ambiente em que vivem. Incluindo o desenvolvimento do próprio ser (auto-estima, sistema de valores, capacidade de análise, energia, diferenciação, capacidade de lidar com o risco, criatividade), levando consigo estas experiências como inspiração para desenvolverem-se profissionalmente e como cidadãos (Gibson, 1997, Dolabela, 2003, Pimenta, 2004).

\section{Contexto e Infra-estrutura}

As experiências descritas a seguir têm sido realizadas na Escola Municipal de Ensino Fundamental Ernani Silva Bruno, Parada de Taipas, município de São Paulo, local no qual trabalha o autor ${ }^{*}$, em reuniões e conferências diversas através de palestras e oficinas, e no Laboratório de Sistemas Integrados - LSI, sob coordenação do autor ${ }^{* *}$, local onde têm sido realizados experimentos educacionais usando o estado da arte da tecnologia através de técnicas de Realidade Virtual no ambiente digital imersivo CAVERNA Digital ${ }^{\mathrm{TM}}$ (LSI, 2004).

\section{Hardware}

As ações relativas à disseminação e uso de novas tecnologias no ambiente escolar vem acontecendo de modo gradativo. Por exemplo, o laboratório de informática que estava equipado até o final de 2002 com 17 computadores interligados por cabos coaxiais, offline, com sistema operacional Windows $95,133 \mathrm{MHz}, 16 \mathrm{RAM}$, agora está configurado com 21 computadores conectados com a Internet. Há dois sistemas operacionais em cada máquina, Windows XP e Linux Conectiva 8.0. A capacidade de processamento das máquinas novas foi ampliada significativamente para $900 \mathrm{Mhz}, 128 \mathrm{RAM}$. A finalização de instalação de conexão com a Internet e os novos computadores tem gerado nos educadores/educandos mais motivação para desenvolver os conteúdos do currículo tanto quanto as diversas habilidades individuais através das TICs.

\section{Software}

O software utilizado é em sua maioria conhecido pelos educadores/educandos o que facilita sua manipulação para desenvolvimento inicial do aprendizado. Além disto são acessíveis mesmo para os indivíduos que não tenham recursos econômicos e computacionais disponíveis em grande quantidade, como no caso da maioria dos educadores/educandos que formam a comunidade escolar brasileira. 
- Cosmo Player 2.1 - browser para visualização e navegação em mundos virtuais 3D construídos em VRML.

- VRML - linguagem para modelar mundos virtuais em 3D.

- HML - para formatação de página WEB.

- Paint - para visualização e criação de imagens.

- GIMP - para visualizar, tratar e otimizar imagens.

- Internet Explorer ${ }^{\mathrm{TM}}$ - navegador

- WordPad - editor de texto

\section{Experimentando com Novas Tecnologias na Formação de Educadores}

Em Julho de 2002 o autor* apresentou para professores do Ensino Fundamental I o mundo virtual por ele construído para que fossem analisadas possibilidades de utilizar tal tipo de instrumento no trabalho de desenvolver as habilidades de leitura e escrita dos alunos, ampliar o acesso às tecnologias digitais e propiciar mais qualidade na formação da comunidade escolar. A mostra ocorreu devido ao interesse dos professores em conhecer o trabalho apresentado pelo autor* na 8th International Consortium Learning Conference - ICEL 2002, na Faculdade de Artes em Ljubljana, Eslovênia (Figura-1).

Segundo [Valente 1993, em Candau 2003] para que estas ações de disseminação tecnológica sejam efetivas é necessário que o processo formação dos professores propicie aos educadores dominar o computador e tecnologias afins para que tenham condições de integrá-los à sua disciplina. A conscientização acontece através do processo de "reflexão na ação e sobre a ação", para que haja mudança de atitudes ou valores de trabalho com entendimento sobre a nova postura que o educando/educador precisa assumir para transformar o processo ensino - aprendizagem, e faça uso efetivo das possibilidades de construção de conhecimentos interativos gerados pelas TICs (Celani, 2003).

Por exemplo, ao inicio da palestra durante a ICEL 2002, um dos professores que iriam assisti-la perguntou ao autor ${ }^{*}$ se o desenvolvimento dela seria tão técnico que não haveria possibilidade de ao final das atividades compreender o assunto. $\mathrm{O}$ autor ${ }^{*}$ iniciou a explanação descrevendo sua formação primeira como professor de línguas e que o interesse pela área mais técnica ocorreu com o tempo de prática docente e a necessidade de acompanhar as mudanças que ocorriam no mundo e estar mais próximo de compreender as linguagens explicitadas pelos alunos em suas falas (Internet, Homepage, chat, animações em 3D). Houve continuidade da apresentação que se tornou oficina interativa tanto no processo oral/teórico quanto no aspecto prático. A platéia interagiu com o mundo virtual praticando o jogo da velha, movimentando a bola na quadra de esportes, ouvindo trecho da música "Jesus, Alegria dos Homens", associada com o sensor de proximidade localizado perto da pirâmide com a cruz representando o ano do Senhor. Houve ainda a construção em tempo real um artefato utilizando os conceitos de reutilização de código e construção de interfaces integrando linguagens e sistemas. Ao final os educadores ficaram satisfeitos com as interações e reflexões feitas sobre algumas das possíveis utilizações de linguagens acessíveis da WEB, ferramentas multimídia e técnicas de Realidade Virtual na educação.

\section{Compartilhando Saberes Com Educandos/Educadores em Contexto}


A interação positiva com os educadores durante a conferência reforçou a idéia e a confiança para investigar se na dinâmica diária da escola seria possível implementar as dinâmicas de criação de mundos virtuais no contexto do ensino fundamental I e criar parâmetro de referência mais preciso quanto às reações dos alunos (positivas e negativas) ao interagirem com as novidades. Levando-se em consideração fatores ambientais que inicialmente poderiam desestimular o desenvolvimento da experiência, tais como: linguagem (os comandos das línguas Markup estão em inglês); tempo reduzido para um estudo mais aprofundado do conhecimento da linguagem utilizada para modelar mundos virtuais 3D; desenvolver o trabalho durante aulas de 45 minutos atendendo às diferentes solicitações dos 36 alunos divididos em grupos realizando alguns outros projetos em paralelo com a implementação da experiência, enquanto o número de estudantes recomendado por estudos para que haja bom atendimento educacional através da Telemática são 20 alunos por educador segundo experiências realizadas por (Valente, 2003), quantidade que acreditamos ser ideal se aplicada como regra na constituição de classes da rede escolar pública em conformidade com as propostas de mudanças para uma educação mais interativa, inclusiva e de qualidade.

\section{Implementação da Experiência e Estratégias}

Participando e observando do desenvolvimento do trabalho realizado com uma classe do $4^{\mathrm{a}}$ ano do ciclo básico I e a movimentação dos alunos na maneira de ilustrarem seus textos usando o programa Paint na criação de um tabuleiro de xadrez e movimentando o cursor na tela como se estivessem jogando, o autor ${ }^{*}$ concluiu que era o momento apropriado para implementação da experiência e desenvolver o projeto. Apresentou para a professora o mundo virtual da Figura -1, as ferramentas necessárias para produção dos arquivos que comporiam a construção do mundo virtual e propôs que os alunos construíssem um jogo virtual com base no enredo da escola Gaviões da Fiel de 2002, cujo tema era "jogo" descrito na letra do enredo Xeque Mate.

A professora concordou prontamente com a idéia. Inicialmente os alunos manipularam o mundo virtual supra e tiveram contato com o exemplo interativo que vem com o browser Cosmo Player 2.1. O autor ${ }^{*}$ consultou-os se aceitariam criar um jogo de xadrez virtual para conhecerem e aplicarem algumas linguagens desenvolvidas para Internet, $o$ que o grupo de alunos aceitou prontamente. Os alunos receberam a explicação que todas as ações seriam realizadas durante a aula e que seria necessário comprometimento na execução da tarefa. Foram feitos os primeiros exercícios e aos poucos todos ganhamos confiança uns nos outros e satisfação pelo desenvolvimento de nossas interações humanas e habilidades técnicas. Para ilustrar, apresentamos a seguir o parecer da professora da sala que participou do projeto e como transcorreu o aprendizado.

"Tabuleiro de Xadrez. A idéia do trabalho surgiu durante a confecção de atividades desenvolvidas para uma amostra cultural na escola. O tema trabalhado foi a Região Sudeste, seus aspectos geográficos, econômicos e culturais. Decidimos ampliar nossos saberes na área cultural abordando o tema carnaval. Os alunos se mostraram bastante interessados e participativos na realização desse trabalho, até mesmo aqueles com mais dificuldades na sala de aula mostraram um bom desempenho. Foi muito positivo o resultado do trabalho, pois os alunos tiveram um ganho na qualidade do raciocínio, além de desenvolver o espírito em trabalhos de grupo. A experiência refletiu de forma significativa na realização de outras atividades em sala de aula no que diz respeito a responsabilidade, autonomia e auto-estima. Os alunos sentiram-se importantes com a 
tarefa delegada a eles, perceberam que eram capazes e que se trabalhassem em grupos de forma organizada o resultado seria melhor e mais rápido."

Helenice de Oliveira - Professora da 4B em 2002 - reflexões descritas em Abril de 2003.

Devido a falta de um tempo maior para estudo e modelagem constante das peças do xadrez não foi possível como previsto terminar todo o processo de construção do artefato, mas o engajamento dos alunos em aprender e explorar novas habilidades é onde está o maior ganho no trabalho realizado. Foram praticados conceitos de reutilização, trabalho cooperativo e colaborativo, atenção e concentração para leitura de código, participação na construção de um protótipo de Homepage utilizando as linguagens HTML e VRML, visualizado off-line na "I EDUCAINFO - Reflexão Para Transformação da Sociedade", encontro organizado pelo Núcleo de Ação Educativa - 4 (NAE-4), ocorrido dia 18/11/2002 na Estação Ciências.

Houve também, mais interação entre os POIEs da escola, pois durante a construção do modelo as crianças detectaram que o código utilizado para construção do tabuleiro apresentava uma casa a menos, e foi preciso estudar juntos e agrupar conhecimentos inter e multidisciplinares (matemática e inglês) para resolver o problema.

\section{Interatividade e fundamentos Pedagógicos}

Interações entre crianças e linguagens de programação orientadas por educadores estão favorecidas pela influência das tecnologias digitais interativas no trabalho, escola, e lares, o que propicia a atual geração de crianças múltiplas oportunidades de interagirem com sistemas eletrônicos. Conforme Masetto (1997), é recomendável que a escola se aproprie e use com efetividade os instrumentos multimídia à disposição na construção do conhecimento de educandos/educadores. As constantes interações com tecnologias diversas têm levado as crianças a navegar em sistemas de complexidade múltipla e criar projetos utilizando tais tecnologias com qualidade muito maior do que os adultos. Por exemplo, o domínio quase inato que demonstram ao jogar vídeo game (Kafai in Druin 1999).

As ações pedagógicas e tecnológicas das experiências em curso estão fundamentadas nas idéias e teorias do Construcionismo de Papert, e construtivismo de Piaget e Dewey exemplificadas também nas ações propiciadas pelo projeto NICE (Dewey, 1966, Piaget, 1973, Parpet, 1980 em Roussus et. al, 1997) contribuindo para o aprendizado ativo dos indivíduos, que têm sua assimilação do conhecimento estimulada ao utilizar e desenvolver suas habilidades, manipulando, explorando, construindo, objetos, sistemas e idéias.

No contexto das transformações sociais necessárias, nas quais a escola pode e deve ter um papel importante, reportamo-nos às idéias de Paulo Freire estudadas por (Gadotti, 1994) e o conceito do desenvolvimento proximal de Vygotsky apresentado no trabalho de (Schwienhorst, 1998) para embasar o pensamento relativo à importância no uso da sinergia tecnologia/educação como agente-chave na autonomia do aprender a aprender de educandos/educadores.

O uso da tecnologia pode incrementar as relações entre educadores e crianças, política e educação, contribuindo para aquisição de conhecimento como relevante fator de melhoria social, proporcionando expressões multi-culturais e integração universal dos 
indivíduos. A padronização de linguagens e protocolos da Internet permite grande mistura e manifestação cultural e social com base em tecnologia de ponta. Assim sendo, a inclusão digital pode ser instrumento efetivo para ampliar o letramento dos indivíduos, estimular a auto-estima com respeito aos diversos aspectos culturais relativos às técnicas, tempo, espaço, razão e emoção (Santos, 1996). Como exemplo, temos a contribuição de âmbito nacional e internacional através das ações educativas estimuladas pela Feira Brasileira de Ciências e Engenharia - FEBRACE, coordenada pelo autor $^{* *}$ (FEBRACE, 2004).

\section{Trabalho Futuro}

Iniciado em setembro de 2003, o trabalho em progresso está norteado pela prática/teoria discutidas no decorrer do texto com estratégias muito similares as descritas anteriormente, mas voltado a orientar os estudantes do ensino fundamental I e II, objetivando prepará-los para compartilhar o conhecimento adquirido com seus pares dando maior escala a disseminação das tecnologias acessíveis que são estudadas durante duas aulas, uma vez por semana durante o horário de estudos do autor ${ }^{*}$.

Antes de iniciar o ciclo de estudos convidamos dez alunos que participavam do projeto Educom.rádio para assistirem uma peça teatral montada com base em tecnologia, cujo nome é "Acordei que Sonhava" produzida pelo Núcleo Bartolomeu de Depoimentos. A idéia era fundamentar melhor através da experiência ativa como os equipamentos multimídia podem contribuir na hora de expressarmos os conteúdos que desejamos e que é preciso preparar-se para isso, procurando conscientizar os jovens estudantes que o êxito de muitos projetos é resultado de muito mais transpiração do que inspiração (Dolabela, 2003).

Com base na prática e teoria do aprendizado experimental utilizada durante as oficinas do projeto Garagem Digital ${ }^{\mathrm{TM}}$, voltado à iniciação tecnológica de adolescentes, no qual o autor ${ }^{*}$ participou ativamente conforme (Franco e Roseli, 2002, Pimenta, 2004, TCL, 2004), foi feito o convite para os estudantes participarem dos momentos de estudos. Seis deles aceitaram. Pesquisamos e aprendemos juntos conceitos relativos às tecnologias acessíveis referenciadas anteriormente no texto até o final de novembro de 2003. Retomaremos os estudos a partir de março de 2004. Um fato importante que engloba o espírito do projeto é a confiança que vai se estabelecendo em cada um de nós no sentido de perseverar e aperfeiçoar-se. Como exemplo, um dia que o autor* precisou sair do laboratório. Ao voltar encontrou mensagem no quadro magnético com o seguinte teor: "professor está difícil, mas tenho certeza que vamos conseguir resolver os problemas" - Emanuely -.

\section{Conclusão}

As ações apresentadas neste trabalho demonstraram que é importantíssimo compartilhar conhecimento técnico e humano para ampliar as formas de acesso dos indivíduos à Educação formal e informal de modo permanente, incluindo o mundo das novas tecnologias para propiciar o bem comum.

Adquirir confiança no que se faz traz o equilíbrio e consciência para realizar transpondo as dificuldades com perseverança. Os ambientes de aprendizagem podem contribuir para o equilíbrio mental e emocional dos indivíduos, através de educação e currículo 
transformadores, fundamentados no conceito de educação dialógica horizontal entre educadores/educandos alimentada pelo amor, humildade, esperança, fé e confiança (Freire, 1967, Gadotti, 1994, Franco e Roseli, 2002a).

A integração das TICs na educação pode efetivamente contribuir para a transformação do contexto escolar, modificando-o para um processo muito mais dinâmico de mudança e melhoria curricular e social. Ampliando por meio do uso de sistemas e conceitos, por exemplo, como os que compõem a realidade virtual, a qual apresenta características interativas e participativas, possibilidades de auto-expressão dos indivíduos.

As observações, experiências e reflexões realizadas até o momento sobre a aplicação das novas tecnologias na educação indicam a viabilidade e validade de continuar procurando formas de escalar para mais pessoas o conhecimento sobre tais tecnologias. Assim sendo, poder contribuir para o desenvolvimento das diversas competências necessárias para que os indivíduos estejam preparados para interagirem com criatividade com os desafios que a sociedade da informação nos apresenta.

\section{Agradecimentos}

Agradecemos aos educadores e educandos que têm gentilmente colaborado com estas investigações, perseverando, criticando construtivamente, sobre tudo nos incentivando a aprender a aprender.

\section{Referências Bibliográficas}

1. Ames, A., L.; Nadeu, D., R.; Moreland, J., L., (1997) VRML 2.0 Sourcebook second edition, Wiley and Sons, USA. Exemplos disponíveis em: www.wiley.com/compbooks/)

2. Biazus, M. C. V. É possível viver o digital na multimídia utilizada com fins educaionais? Disponível em: www.cinted.ufrgs.br/renote/fev.2003/index.htm

3. Candau, M Vera (2003) A configuração de um novo Educador, (www.dhtnet.org.br/direitos/militantes/veracandau/candau_novoeducador.html)

4. Castells, M. (2003) A Galáxia da Internet: reflexões sobre a Internet, os negócios e a sociedade, editor JZE, tradução Maria Luiza X. de A, Borges, Rio de Janeiro.

5. Celani, M., A., A., (ORG.) (2003) Professores e Formadores em mudança: Relato de um Processo de Reflexão Transformação da Prática Docente, Mercado de Letras, São Paulo, Brasil.

6. Dolabela, F. (2003) Pedagogia Empreendedora, Editora Cultura, Brasil.

7. Doll, W. E. Jr. (1997) Currículo: uma perspectiva pós-moderna, tradução. Maria Adriana V. V., Artes Médicas, Porto Alegre.

8. Durin, Alison (1999) The Design of Children's Technology, Morgan Kaufmann Publishers, San Francisco, USA.

9. FEBRACE - FEIRA BRASILEIRA DE CIÊNCIAS E ENGENHARIA WWW.LSI.USP.BR/FEBRACE

10. Franco, J. F. Multimedia in Action: Applying 3D environments at school teaching, using VRML for an interactive, dynamic and high quality education. GEMISIS Conference Digest, University of Salford, UK, 2000.

11. Franco, J. F. Teaching and Learning Using Interactive 3D Virtual Environments and Multimedia tools, International Consortium of Experiential Learning (2000a), The University of Auckland, New Zealand.

12. Franco. J.F., Lopes R. D. - From children to Adults: Improving Education through Virtual Environments Technologies, Proceedings of the $5^{\text {th }}$ IASTED International Conference Computers Advanced Technology in Education May 20 - 22, 2002, Cancun, Mexico. 
13. Franco. J.F., Lopes R. D. - Lifelong Learning to Learn with Arts and Interactive 3D Graphics - Proceedings of the 8th International Consortium of Experiential Learning (2002a), at the University of Ljubliana, Faculty of Arts in Slovenia, 1 to 5 July.

14. Freire, P., (1967). Educação como prática da liberdade (Education as practice of freedom. Paz e Terra, Rio de Janeiro.

15. Gadotti, M., (1994) Reading Paulo Freire, his life and work, Translated by John Milton, State University of New York Press.

16. Gibson, R. (1997) Rethinking the Future, NB, London, UK.

17. LSI - LABORATÓRIO DE SISTEMAS INTEGRADOS ( www.lsi.usp.br/ rv).

18. Masseto, Marcos (1997). Didática: a aula como centro. FTD, São Paulo.

19. Perlin, K., Home page, Disponível em : http://mrl.nyu.edu/ perlin (2004)

20. Pimenta, F., Associação Meninos do Morumbi, Disponível em http://www.meninosdomorumbi.org.br/entrada.php .

21. Roussos, M.; Andrew E.; Leigh, J., Barnes, C. R.; Vasilakis, C., A.; Moher T. G., The NICE project: Narrative, Immersive, Constructionist/Collaborative Environments for Learning in Virtual Reality,University of Illinois at Chicago, (1997), Disponível em http://www.ice.eecs.uic.edu/ nice/

22. Santos, N., (1996) A natureza do espaço, técnica e tempo, razão e emoção, HUCITEC, Brasil.

23. Schwienhort, K. (1998) Co-constructing learning environments and the learner identitieslanguage learning in virtual reality. Proceedings of the ED-Media/ED-Telecom, Freiburg.

24. Valente, A., J., (Org.) (2003) Formação de Educadores para o Uso da Informática na Escola. UNICAMP/NIED, Campinas. 